\title{
Sobre a Entamoeba brasiliensis
}

\author{
pelo
}

\author{
DR. HENRIQUE DE BEAUREPAIRE ARAGÃO
}

(Assistente).

(Com a estampa 1.)

\section{Ueber Entamoeba brasiliensis}

\author{
von \\ DR. HENTIQUE DE BEAUREPAIRE ARAGÃO, \\ Assistent am Institute.
}

(Mit Taf. 1.)

Já tivemos ocasião de descrever resumidamente esta entameba, parasito do intestino humano, em pequena nota, ha algum 6 tempo publicada no "Brazil Medico" (N. 7 de $15-12-912$ ) e que hoje podemos ampliar com estude mais completo de sua mor- folojia e malpr numero de desenhos dos seus diferentes aspetos.

A Entamoebr $\bullet$ rasiliensis foi encontrada nas fezes solidas de criança muito anemica, e por isso, suspeitada de sofrer de anquilostomiase.

Feita a pesquiza microscopica só encontrámos no material examinado raros ovos de tricocefalo, sendo, porém, nele muito numerosas as entamebas, que nos ocupam, em faze de encistamento e raras em periodo vejetativo e estas
Diese Entamoebe, welche den menschlichen Darm bewohnt, habe ich bereits kurz in einer Mitteilung beschrieben, welche im «Brazil-Medico» N. 7 vom 15ten Jan. 1912 erschien; heute kann ich diese Beschreibung durch ein genaueres Studium ihrer Morphologie und eine groessere Zahl von Abbildungen ihrer verschiedenen Formen ergaenzen.

Die Entamoeba brasiliensis wurde in den konsistenten Faeces eines, wegen starker Anaemie der Ankylostomiasis verdaechtigen, Kindes gefunden.

Bei der mikroskopischen Untersuchung fanden wir in den Faezes nur vereinzelte Eier von Trichocephalus, waehrend die uns beschaeftigende Entamoebe sehr zahlreich darin vorhay den war. Sie fand sich hauptsaechlich 
mesmas em fase de preencistamento e imoveis.

As tentativas feitas para se obter formas vejetativas abundantes pela administração de purgativos á doente, que depois perdemos de vista, não surtiram efeito.

Com as fezes ricas de entamebas foram alimentados e injetados por via retal alguns gatinhos, sem que, porém, conseguissemos infetal-os.

Ao exame microscopico, a fresco, do material, eram facilmente reconheciveis as entamebas sob a forma de pequenas massas arredondadas, pouco refrinjentes, tendo no interior um nucleo, pequenos vacuolos e inclusões. Nos cistos se distinguiam sem dificuldade a membrana, os nucleos, as massas cromidiais e filamentos de tamanho diverso que logo nos prenderam a atenção.

Mais instrutivo, porém, a respeito da estrutura do protozoario é, certamente, o seu estudo em preparados fixados e córados, pois que então os seus caracteres morfolojicos se tornam perfeitamente evidentes.

Para o exame do material, nestas condições, recorremos, com vantajem, á fixação humida dos esfregaços em laminulas, e ulterior coloração pela hematoxilina ferrea de HEIDENHAIN. O borax carmim e a hematoxilina de DELAFIELD oferecem resultados menos favoraveis e não permitem tão clara diferenciação dos elementos estruturais do protozoario, como a que se consegue com o processo acima citado.

No estádio vejetativo, em fase de preencistamento, apresenta-se a Entamaba brasiliensis sob a forma de massas arredondadas de 10 a $15 \mu$ de diametro.

O protoplasma é finamente alveolar, com vacuolos nutritivos e pequenas inclusões, principalmente constituidas por bacterios $\mathrm{e}$ residuos da alimentação.

O nucleo é esferico, mede 3 a $4 \mu$ de eixo e tem por limite extremo uma membrana delgada, á qual se aplicam pequenas massas de cromatina. No seu interior vêm-se uma zona de suco nuclear e filamentos acromaticos e no centro um pequeno cariosoma, em cujo interior não nos foi possivel 'cistin- inzystiert und nur selten in der vegetativen Periode, aber auch dann unbeweglich und in Vorbereitung zur Zystenbildung.

Versuche, reichlichere vegetative Formen durch Abfuehrungsmittel zu erzielen, gaben kein Resultat; auch verloren wir spaeter die Patientin aus den Augen.

Die an Entamoeben reichen Faezes wurden jungen Katzen per os und per rectum beigebracht, ohne das es gelang eine Infektion herbeizufuehren.

Bei mikroskopischer Untersuchung des frischen Materiales liessen sich die Entamoeben leicht in Form kleiner rundlicher und wenig lichtbrechender Massen erkennen, welche in ihrem Innern einen Kern, kleine Vakuolen und Einschluesse zeigten. An den Zysten erkannte man leicht die Membran, die Kerne, die Chromidialmassen und Filamente verschiedener Groesse, welche sofort meine Aufmerksamkeit erregten.

Beim Studium der Struktur dieses Organismus sind jedoch fixierte und gefaerbte Praeparate entschieden weit lehrreicher, da dann seine morphologischen Eigenthuemlichkeiten vorzueglich hervortreten.

Zur Untersuchung des Materiales in dieser Form bediente ich mit Vorteil der feuchten Fixierung von Ausstrichen auf Objekttraegern und nachtraeglicher Faerbung mit Eisenhaematoxylin nach HEIDENHAIN. Boraxkarmin und DELAFIELDsches Haematoxylin geben weniger guenstige Resultate und gestatten keine so deutliche Darstellung der Einzelheiten der Struktur, wie man sic durch das angefuehrte Verfahren erzieit.

Im vegetativem Stadium, kurz vor der Inzystierung, bildet die Entamoeb $\imath$ brasiliensis rundliche Massen von $10-15 \mu$ Uurchmesser. Das Protoplasma ist feipwabig und zeigt Ernaehrungsvakuolen neben kleinen Einschluessen, welche hauptsaechlich aus Bakterien und Nahrungsresten bestehen.

Der Kern ist kugelig, 3-4 $\mu$ im Durchmesser, und nach aussen von einer feinen Membran begrenzt, welcher kleine Chromatinmassen anliegen. Im Innern sieht man eine Kernsaftzone, achromatische Filamente und im Zentrum ein kleines Karyosom, in 
guir a presença de centriolo. (Est. 1, fig. 1).

Logo ao começo do encistamento não existe membrana em torno das entamebas; porém ela não tarda a aparecer muito distinta, hialina e duplamente contornada. O protoplasma nos cistos é mais condensado do que nas formas vejetativas e, com exceção dos cromidios, geralmente desprovidos de inclusões ou em via de se libertar delas.

E' excepcional nos cistos mono e binucleados da Entamœeba brasiliensis a presença de grandes vacuolos e tambem somente raras vezes conseguimos, ainda, encontrar no protoplasma do protozoario encistado, massas acinzentadas. lembrando substancia de reserva. (Est. 1, fig. 2).

O numero de nucleos no cisto varia de 1 a 8 e em geral são eles tanto menores, quanto mais abundantes, ao passo que o aumento de volume total do cisto é proporcional ao numero de nucleos nele existentes. Assim, os cistos que têm até 4 nucleos, medem 7 a $10 \mu$ e 12 a $15 \mu$ quando o numero de nucleos vae de 5 a 8 .

$\mathrm{O}$ aspeto morfolojico do nucleo dos cistos em repouso é muito semelhante ao das fórmas vejetativas, somente a cromatina apresenta-se sob a fórma de pequenas massas mais isoladas da membrana do que nas fórmas vejetativas.

As fases de divisão dos nucleos nos cistos são extremamente raras e as suas minucias só podem ser bem observadas nas primeiras divisões nucleares, porquanto nas alteriores a pequenhez do objeto não permite observar-lhe a estrutura fina.

O prucesso de divisão nuclear no cisto se faz, como judemos observar, segundo o tipo de mitose descrito primeiram ente por PROWAZEK para a Entarioeba buccalis, com a formação de pequéno fuso constituido pelos elementos do cariosoma, independente da divisão do nucleo exterior, cuja cromatina se dispõe ao longo da membrana ; finalmente, a massa nuclear se alonga e se divide ao centro sem formação por sua parte de fuso tipico (Est. 1, fig. 2). A extrema variedade das fases de divisão torna dificil o seu estudo com maiores minucias. A' proporção que os dessen Innern ich kein Zentriol wahrnehmen konnte (Taf. 1, Fig. 1).

$\mathrm{Zu}$ Beginn der Inzystierung sind die Entamoeben von keiner Membran umschlossen, sie erscheint indessen bald sehr deutiich, hyalin und doppelt kontouriert. Das Protoplasma ist in den Zysten dichter, als in den vegetativen Formen und bis auf die Chromidien von Einschluessen frei oder im Begriff sich von denselben zu befreien.

In ein-und zweikernigen Zysten der Entamoeba brasiliensis ist das Vorkommen von grossen Vakuole:1 ungewoehnlich; ebenso sah ich im Protoplasma der Zysten nur selten graue Massen, welche an Reservesubstanzen erinnerten (Taf. 1, Fig. 2).

Die Zahl der Kerne einer Zyste variirt von 1-8; gewoehnlich sind sie um so kleiner, je zahlreicher sie sind, waehrend die Groesse der Zyste der Anzahl der darin enthaltenen Kerne proportional ist. So messen Zysten mit hoechstens vier Kernen 7-10 $\mu$ und $12-15$, wenn die $\mathrm{Zahl}$ derselben fuenf bis acht betraegt.

Morphologisch ist der ruhende Kern der Zy'sten dem der vegetativem Formen sehr aehnlich, nur erscheint das Chromatin in der Form kleiner Massen, welche von der Membran mehr abstehen, als bei den vegetativen Formen. Die Kernteilungsphasen sind in den Zysten sehr selten und ihre Einzelheiten koennen nur bei den ersten Teilungen gut beobachtet werden, da bei den weiteren die Kleinheit des Objektes eine genauere Beobachtung hindert.

Wie ich verfolgen konnte, vollzieht sich die Kernteilung in der Zyste nach dem $\mathrm{Mi}$ tosentypus, der zuerst von PROWAZEK bei Entamoeba buccalis beschrieben wurde, unter Bildung einer kleinen Spindel, welche aus den Elementen des Karyosoms best $\mathrm{h}$ t, unabhaengig von der Tellung des Aussenkernes, dessen Chromatin sich laengs der Membran ordnet; endlich streckt sich die Kernmasse und teilt sich in der Mitte, ohne ihrerseits eine typische Spindel zu bilden (Taf. 1, Fig. 2). Die grosse Mannigfaltigkeit der Teilungsphasen erschwert ein eingehenderes Studium.ein dem Masse, wie die Zysten ihre Ent- 
cistos completam a sua evolução, vão deles desaparecendo as inclusões e vacuolos nutritivos, somente permanecendo no interior, ás vezes, até o fim, os chamados cromidios, cristaloides ou formações siderofilas. Estas formações se apresentam na Entamoeba brasiliensis sob dois aspetos diferentes, ora com a forma de massas, ora como filamentos ou faixas muito estreitas.

As massas cromidiais não diferem em aspeto das demais formaçõis analogas encontradas em outros protozoarios do grupo, principalmente na Entamoeba tetragena e menos frequentemente na Entamoeba coli. (Est. 1, fig. 2, 4, 6). Quanto ás faixas e filamentos siderofilos eles são carateristicos e logo chamam a atenção de quem observa a entameba. São encontrados no protozoario, ora sós (Est. 1, fig. 3, 7, 8, 9, 10, 11, 12), ora em companhia das massas cromidiais acima citadas e em qualquer estádio de evolução do cisto. (Est. 1, fig. 3 e 5 a 13).

Estes filamentos siderofilos são geralmente duplos (Est. 1, fig. 7, 8, 9, 12, 13), ao começo eles são representados nos cistos por finos filamentos (Est. 1, fig. 8.) que aos poucos, se vão tornando mais espessos do centro para a periferia (Est. 1, fig. 7, 9, 10). O sou tamanho varia, ora se reduzem esses cromidios a filamentos pequenos estendidos ou entortilhados em pequena rejião do cisto, outras vezes a disposição deles é sob a forma de longos fios que váo duma parede a outra do cisto, dividindo-o em partes quasi iguais (Est. 1, fig. 12). Esta disposição dos filamentos paraceu-nos a principio estar relacionada com uma manifestação sexual, dando-se no cisto a separação dos nucleos das amebas que ulteriormente deveriam copular ao sair dele. $\mathrm{O}$ estudo ulterior do nosso material, nos fez modificar essa opinião que não nos parece hoje suficientemente baseada. Nenhuma hipotese, por isso, queremos emitir sobre o papel dos chamados cromidios filamentosos na Entamoeba brasiliensis, nem a razão de ser da sua disposição no interior dos cistos.

A frequencia desses cromidios filamentosos, assim como a abundancia das massas wicklung vollenden, verschwinden aus denselben die Einschluesse und Naehrvakuolen, indem darinnen, manchmal bis zum Schlusse, die sogenannten Chromidien, Krystalloide und siderophile Bildungen verbleiben. Diese Gebilde erscheinen in der Entamoeba brasiliensis in zweierlei Form, entweder als Massen oder als sehr schmale Fasern und Buendel.

Die Chromidialmassen unterscheiden sich ihrem Aussehen nicht von analogen Formen, wie sie bei anderen Arten aus derselben Gruppe, beonders bei Entamoeba tetrigenae und seltener bei Entamoeba coli beobachtet werden (Taf. 1, Fig. 2,4,6). Dagegen sind die siderophilen Fasern und Buendel charakteristisch und fesseln sofort die Aufmerksamkeit des Beobachters. Man findet sie bald allein (Taf. 1, Fig. $3 \& 7$-12), bald neben den oben erwaehnten Chromidialmassen und zwar in allen Stadien der Zysten (Taf. 1, Fig. $385-13$ ).

Diese siderophilen Fasern sind gewoehnlich doppelt (Taf. 1, Fig. 7-9, 12 \& 13); zuerst erscheinen sie in den Zysten als feine Filamente (Taf. 1, Fig. 8), welche sich nach und nach von der Mitte nach den Enden zu verdicken (Taf. 1, Fig. 7, $9 \&$ 10). Ihre Laenge schwankt; bald beschraenken sie sich auf kleine, gestreckte oder eingerollte, Fasern in einem kleinen Teile der Zyste, andere Male erstrecken sie sich in Gestalt langer Faeden von einer Wand zur andern und teilen die Zyste in zwei annaehernd gleiche Teile (Taf. 1, Fig. 12). Diese Anordnung der Fasern schien mir urspruenglich auf einen geschlechtlichen Vorgang zu deuten, bei welchem is Innern der Zyste eine Trennung der Kegne stattfaende, welche spaeter nach dem Av stritt aus der Zyste kopulieren wuerden, allein weitere Str dien an meinem Materiale diessen mir Gies Auffassung nich genuegend begruendet $\mathrm{cr}$ scheinen. Ich will daher ueber die Rolle der faserigen Chromidien und ueber die Ursache ihrer Anordnung in den Zysten der Entamoeba brasiliensis keine Hypothese aufstellen.

Die Haeufigkeit dieser faserigen Chromidien und die Menge der Chromidialmassen, im Verein mit den kleinen Dimensionen, sind nach meiner Auffassung, fuer die Entamoeba 
cromidiais, junto ás pequenas diniensões da Entamoeba brasiliensis a tornam, a nosso ver, perfeitamente carateristica e distinta da Entamoeba coli.

Não podemos estar de acordo com os protozoolojistas que querem considerar todas as amebas com 8 ou mais nucleos encontradas no intestino humano, como sendo Enta- moeba coli, pois, a nosso ver, muitas delas têm caracteres bastante distintos uma das outras, si bem que com aspeto geral semelhante. Tambem, a principio, ninguem pensava em separar as amebas limax e mais tarde se verificou, quão grande é o numero de especies existentes nesse grupo e, até mesmo, generos foram nele separados. Estamos certo do que o mesmo acontecerá em relação ás entamebas do grupo coli, pois nos custa aceitar que as diferenças morfolojicas, já assinaladas para um certo numero de protozoarios desse grupo, possam ser consideradas como simples variações individuais, tal a fixidez dos seus caracteres.

Manguinhos, Janeiro, 1914. brasiliensis ganz charakteristisch und unterscheiden dieselbe genuegend von der Entamoeba coli.

Ich kann den Protozoenforschern nicht beipflichten, welche alle Amoeben mit acht oder mehr Kernen, die im menschlichen Darmkanale gefunden werden, als Entomoeba coli ansehen, da, meines Erachtens, viele derselben unter einander deutliche Unterschiede zeigen, obwohl sie sich im Ganzen und Grossen aehnlich sehen. So hat auch anfaenglich niemand daran gedacht, die Amoeben vom Limaxtypus zu trennen und doch sah man spaeter ein, wie gross die Anzahl der Arten in dieser Gruppe ist und trennte sogar neue Gattungen von derselben ab. Ich bin ueberzeugt, dass dasselbe mit den Entamoeben aus der Coligruppe geschehen wird, da mir schwer wird, zu glauben, dass die morphologischen Unterschiede, welche bereits fuer eine gewisse Anzahl dieser Amoeben hervorgehoben wurden, nur als individuelle Variationen gelten sollten, weil die Bestaendigkeit ihrer Charaktere dafuer viel $\mathrm{zu}$ gross ist.

Manguinhos, Januar 1914. 


\section{Explicação da Estampa 1.}

Material fixado pelo sublimado alcool e corado pela hematoxilina ferrea de HEIDENHAIN.

Desenhos feitos com a camara clara, á altura da mesa, com a ocular compensadora 18 e objet. apocromatica de $2 \mathrm{~mm}$. ZEISS.

Fig. 1. Entamoeba brasiliensis: forma vejetativa em periodo de preencistamento.

* 2. 1a Divisão nuclear, de massas cromidiais e substancia de reserva(?)

* 3. Cisto mononucleado, longo filamento siderofilo, pequenas inclusões em um vacuolo (cromidios).

- 4. Cisto mononucleado, com uma grande massa cromidial e outras menores.

* 5. Cisto com nucleo grande e dois pequenos, uma massa e um filamento siderofilo.

- 6. Cisto com 4 nucleos e uma massa cromidial.

- 7. Cisto com 3 nucleos e longos filamentos siderofilos, espessados no centro.

4 8. Cisto com 4 nucleos e 2 longos filamentos siderofilos separados.

«98 10. Cisto com 4 nucleos e 2 filamentos siderofilos separados e mais espessados no centro.

11. Cisto com 7 nucleos e 2 longos filamentos siderofilos equatorialmente colocados.

* 12 \& 13. Cistos com 8 nucleos, massas e filamentos cromidiais.

\section{Erklaerung der Tafel.}

Fixierung des Materiales mit Sublimatalkohol und Faerbung mit HEIDENHAINschem Haematoxylin.

Zeichnungen mit Camara lucida auf der Hoehe des Tisches entworfen. Compensationsocular 18 und apochrom. Obj. 2 Mm., ZEISS.

Fig. 1. Entamoeba brasiliensis: Vegetative * Form vor der Inzystierung.

* 2. Erste Teilung von Kern, Chromidialmassen und Reservesubstanz?

3. Mononucleaere Zyste, lange siderophile Faser, kleine Einschluesse in einer Vakuole (Chromidien?).

4 4. Mononukleare Zyste mit einer grossen und mehreren kleineren Chromidialmasse.

5. Zyste mit einem grossen und zwei kleinen Kernen, einer Masse und einer siderophilen Faser.

6. Zyste mit vier Kernen und einer Chromidialmasse.

a 7. Zyste mit drei Kernen und langen siderophilen Fasern, welche in der Mitte verdickt sind.

8. Zyste mit 4 Kernen und zwei getrennten langen siderophilen Fasern.

" $9 \& 10$. Zysten mit vier Kernen und zwei getrennten siderophilen Fasern, die in der Mitte verdickt sind.

- 11. Zyste mit 7 Kernen und zwei langen, aequatorial liegenden, siderophilen Fasern.

"12\&13. Zysten mit acht Kernen, sowie Chromidialmassen und -fasern. 

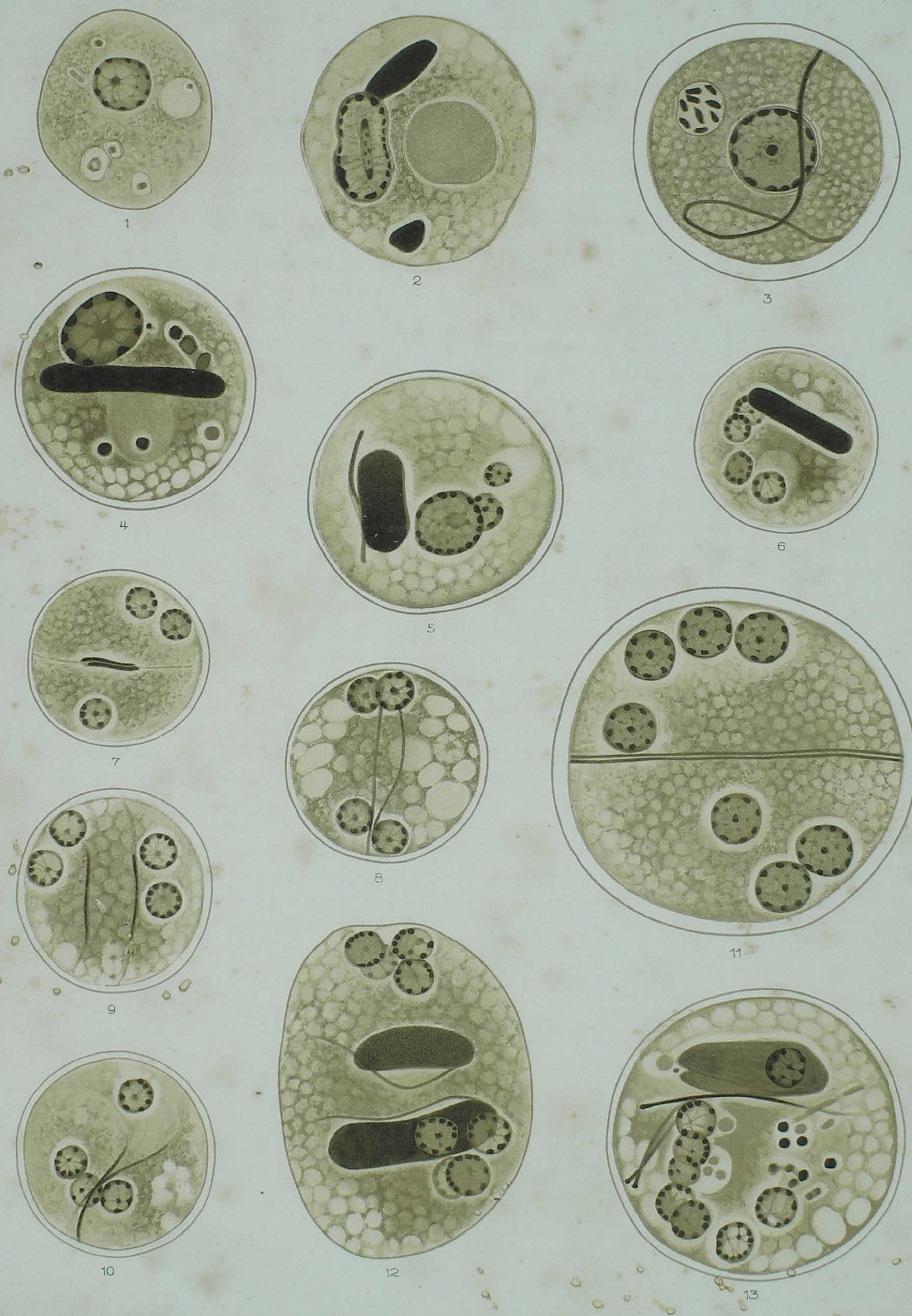